\title{
Parameter Estimations for the Cerebrospinal Fluid Infusion Test
}

\author{
AlMUt EISENTRÄGER*, IAN SOBEY \\ Mathematical Institute, University of Oxford, \\ 24-29 St Giles', Oxford OX1 3LB, UK
}

AND

MAREK CZOSNYKA

Department of Clinical Neurosciences, School of Clinical Medicine, Addenbrookes Hospital, Cambridge CB2 0SP, UK

\begin{abstract}
We consider a single compartment model for a cerebrospinal fluid infusion test using first, an inverse power law cerebral compliance with constant elastance and second, a more recently developed compliance model with elastance depending on intracranial pressure (ICP). A least squares optimisation is used to solve the inverse problem of estimating parameter values from ICP observed during an infusion test. The optimisation is applied to synthetic data and to clinical ICP data from eleven infusion tests. From consideration of synthetic data we show that it is not, in general, possible to distinguish between compliance models and attempting to extract parameter estimates for an extended compliance model is not well posed with different parameter sets having ICP time dependence that cannot be distinguished using current ICP measurements. This conclusion is also confirmed in examination of clinical data.
\end{abstract}

Keywords: cerebrospinal fluid; infusion test; compartment model; parameter estimations.

\section{Introduction}

Abnormal cerebral conditions relating to cerebrospinal fluid (CSF) are very difficult to characterise. One test that can be carried out to evaluate CSF function, particularly for communicating hydrocephalus, is an infusion test (Czosnyka et al. (2004), Iuniewicz et al. (2005)) whereby the usual production rate of CSF in the

*Email: eisentraeger@maths.ox.ac.uk 


\section{A. EISENTRÄGER, I. SOBEY AND M. CZOSNYKA}

choroid plexus is artificially increased for a fixed time period by steady pumping of a CSF-like fluid into the CSF system, usually via a lumbar puncture. During an infusion test the increased CSF 'production' rate results in the CSF intracranial pressure (ICP) rising in order to accommodate the additional fluid and a corresponding increase in drainage, the drainage rate being proportional to the difference between intracranial pressure and pressure in the superior sagittal sinus. In a successful test the intracranial pressure will plateau before the infusion is completed. In some tests the infusion is stopped because of high ICP and a plateau value is not reached. The difference between the original average intracranial pressure and the new average plateau value provides a direct measurement of the resistance to outflow and the rate of rise of the intracranial pressure to the plateau value provides an estimate of cerebral CSF compliance. See for example Marmarou et al. (1978), Czosnyka et al. (2004), Iuniewicz et al. (2005) and Andersson et al. (2008).

A single compartment model for an infusion test balances rate of change in CSF volume against inflow and outflow. In the model used by Iuniewicz et al. (2005) the system is completed once the compliance of the CSF compartment is related to ICP by an inverse power law relation. This allows the compliance to decrease as ICP increases, which is in line with clinical observations. There has been no established theory for what reference pressure should be used, amongst suggested values are zero, the outlet blood pressure and the base ICP pressure (Czosnyka et al., 2004). In Wirth and Sobey (2008) a new model for cerebral compliance was developed by considering changes in blood volume associated with partial collapse of bridging veins. This allows the elastance to vary with ICP and the model used by Iuniewicz et al. (2005), for which the elastance is constant, is a special case of this theory. There is some evidence from clinical measurements that the elastance may vary with pressure, see for example the discussion in Wirth and Sobey (2008). Throughout this paper we will use the phraseology 'standard model' when using the compliance model in Iuniewicz et al. (2005) and 'generalised model' when the compliance is that from Wirth and Sobey (2008).

In this paper we consider the problem of estimating model parameters from observed ICP during infusion tests. We have examined data from eleven clinical tests and have used a least squares minimisation in order to determine parameter values using both the standard model and the generalised model of Wirth and Sobey (2008). The standard model, which can use an analytic solution to a governing differential equation, results in prediction of resistance, base ICP and elastance (and through these the normal CSF production rate). The generalised model, which requires numerical solution of a governing differential equation, provides estimation of these parameters and an additional parameter. Details are given below.

The layout of this paper is that in section 2 we summarise details of the governing differential equation for ICP variation with time and models for cerebral compliance. In section 3 we consider solutions of the governing differential equations and study how prediction of mean ICP is affected by changes in the various governing parameters. In section 4 we outline the methodology for es- 
timating optimal values of parameters from data and in section 5 we consider estimating parameters from synthetic data (that is, known parameter values are used to generate data which is then subject to parameter estimation using least squares optimisation) before applying the optimisation method to clinical data in section 6. Our conclusions are set out in section 7

\section{Theoretical Models}

\subsection{Model Derivation}

We use a single compartment model that treats all the CSF in and around the brain as being in one compartment without spatial variations. Oscillations on the time scale of the heart rate are neglected and all quantities represent an average over a cardiac cycle. Since CSF is water like and incompressible, conservation of CSF mass is equivalent to the conservation of CSF volume, $V$, so that

$$
\frac{\mathrm{d} V}{\mathrm{~d} t}=Q_{\text {in }}-Q_{\text {out }}
$$

where the inflow rate is given as

$$
Q_{\text {in }}= \begin{cases}Q_{\text {prod }}, & \text { before and after the infusion, } \\ Q_{\text {prod }}+Q_{\text {inf }}, & \text { during the infusion, }\end{cases}
$$

and the outflow rate as

$$
Q_{\text {out }}=\frac{p-p_{\mathrm{ss}}}{R}
$$

with a constant CSF production rate $Q_{\text {prod }}$, constant infusion rate $Q_{\text {inf }}$, intracranial CSF pressure (ICP) $p$, venous blood pressure $p_{\mathrm{ss}}$ in the superior sagittal sinus and constant resistance $R$ to outflow through the arachnoid villi.

CSF volume change is linked to ICP via a pressure dependent compliance

$$
C(p)=\frac{\mathrm{d} V}{\mathrm{~d} p},
$$

so that the fluid balance (2.1) yields

$$
C(p) \frac{\mathrm{d} p}{\mathrm{~d} t}=Q_{\text {in }}-\frac{p-p_{\mathrm{ss}}}{R} .
$$

In the time before the infusion begins, oscillations at heart rate being neglected, a steady state with a baseline pressure $p_{\mathrm{b}}$ is assumed, giving

$$
0=Q_{\text {prod }}-\frac{p_{\mathrm{b}}-p_{\mathrm{ss}}}{R},
$$




\section{A. EISENTRÄGER, I. SOBEY AND M. CZOSNYKA}

which allows the unknown production rate $Q_{\text {prod }}$ and pressure $p_{\mathrm{ss}}$ in the sagittal sinus to be eliminated by introducing $p_{\mathrm{b}}$ into the equation to obtain

$$
C(p) \frac{\mathrm{d} p}{\mathrm{~d} t}=Q-\frac{p-p_{\mathrm{b}}}{R}
$$

with initial condition $p\left(t_{1}\right)=p_{\mathrm{b}}$ and

$$
Q= \begin{cases}0, & \left(t_{1} \leq t \leq t_{2}\right) \\ Q_{\text {inf }}, & \left(t_{2}<t \leq t_{3}\right) \\ 0, & \left(t_{3}<t \leq t_{4}\right)\end{cases}
$$

where $t_{1}$ is the beginning of the test (on occasion, data is not usable until some time $\left.t_{1}>0\right), t_{2}$ the start of the infusion, $t_{3}$ the end of the infusion and $t_{4}$ the end of the test.

\subsection{Compliance: Standard Model}

The standard model for the compliance used in Juniewicz et al. (2005) is

$$
C(p)=\frac{1}{E\left(p-p_{\mathrm{r}}\right)}
$$

with an elastance coefficient $E$ and a reference pressure $p_{\mathrm{r}}$. The role of the latter has not been fully understood and as noted above, different values have been suggested for $p_{\mathrm{r}}$, including zero and the venous outflow pressure (Czosnyka et al., 2004). Wirth and Sobey (2008) suggested an alternate value based on mechanical properties of the bridging veins and blood viscosity. Inserting (2.9) into the ordinary differential equation (2.7), it can be reformulated as

$$
p^{\prime}(t)= \begin{cases}-\frac{E}{R}\left(p(t)-p_{\mathrm{r}}\right)\left(p(t)-p_{\mathrm{b}}\right) & \left(t_{1} \leq t \leq t_{2}\right), \\ -\frac{E}{R}\left(p(t)-p_{\mathrm{r}}\right)\left(p(t)-\left(p_{\mathrm{b}}+R Q_{\mathrm{inf}}\right)\right) & \left(t_{2}<t \leq t_{3}\right), \\ -\frac{E}{R}\left(p(t)-p_{\mathrm{r}}\right)\left(p(t)-p_{\mathrm{b}}\right) & \left(t_{3}<t \leq t_{4}\right),\end{cases}
$$

which, with the initial condition $p\left(t_{1}\right)=p_{\mathrm{b}}$, yields the solution

$$
p(t)= \begin{cases}p_{\mathrm{b}} & \left(t_{1} \leq t \leq t_{2}\right), \\ p_{\mathrm{r}}+\frac{\left(p_{\mathrm{b}}-p_{\mathrm{r}}\right)\left(R Q_{\text {inf }}+p_{\mathrm{b}}-p_{\mathrm{r}}\right)}{\left(p_{\mathrm{b}}-p_{\mathrm{r}}\right)+R Q_{\text {inf }} \exp \left(-\frac{E}{R}\left(R Q_{\text {inf }}+p_{\mathrm{b}}-p_{\mathrm{r}}\right)\left(t-t_{2}\right)\right)} & \left(t_{2}<t \leq t_{3}\right), \\ p_{\mathrm{r}}+\frac{\left(p_{\mathrm{b}}-p_{\mathrm{r}}\right)}{1+D \exp \left(-\frac{\left.E_{\mathrm{r}}\left(p_{\mathrm{b}}-p_{\mathrm{r}}\right)\left(t-t_{3}\right)\right)}{1+t_{3}}\right.} & \left(t_{3}<t \leq t_{4}\right) .\end{cases}
$$

The parameter

$$
D=\frac{p_{\mathrm{b}}-p_{\mathrm{r}}}{p\left(t_{3}\right)-p_{\mathrm{r}}}-1
$$

establishes continuity of ICP at time $t_{3}$, the end of the infusion. 
Although not always commented on, it is straight forward once the resistance and ICP are known to calculate the increase in CSF fluid volume during the test, and particularly the maximum increase in CSF volume, expected at the end of the infusion, by

$$
\begin{aligned}
\Delta V(t) & =\int_{t_{1}}^{t}\left(Q_{\text {in }}(s)-Q_{\text {out }}(s)\right) \mathrm{d} s=\int_{t_{1}}^{t}\left(Q_{\text {prod }}+Q(s)-\frac{p(s)-p_{\mathrm{r}}}{R}\right) \mathrm{d} s \\
& =\int_{t_{1}}^{t}\left(Q(s)-\frac{p(s)-p_{\mathrm{b}}}{R}\right) \mathrm{d} s .
\end{aligned}
$$

\subsection{Compliance: Generalised Model}

Wirth and Sobey (2008) propose a generalisation of the compliance (2.9) with a pressure dependent elastance

$$
E(p)=\tilde{e}\left(p-p_{\mathrm{r}}\right)^{n-1},
$$

so that

$$
C(p)=\frac{1}{E(p)\left(p-p_{\mathrm{r}}\right)}=\frac{1}{\tilde{e}\left(p-p_{\mathrm{r}}\right)^{n}}
$$

with an unknown power $n$. Note that the dimension of $\tilde{e}$ depends on the power $n$

$$
[\tilde{e}]=\frac{\mathrm{mmHg}^{(1-n)}}{\mathrm{m}^{3}} .
$$

This model gives a non-linear ordinary differential equation for ICP, $p$,

$$
p^{\prime}(t)= \begin{cases}-\frac{\tilde{e}}{R}\left(p(t)-p_{\mathrm{r}}\right)^{n}\left(p(t)-p_{\mathrm{b}}\right) & \left(t_{1} \leq t \leq t_{2}\right), \\ -\frac{\tilde{e}}{R}\left(p(t)-p_{\mathrm{r}}\right)^{n}\left(p(t)-\left(p_{\mathrm{b}}+R Q_{\mathrm{inf}}\right)\right) & \left(t_{2}<t \leq t_{3}\right), \\ -\frac{\tilde{e}}{R}\left(p(t)-p_{\mathrm{r}}\right)^{n}\left(p(t)-p_{\mathrm{b}}\right) & \left(t_{3}<t \leq t_{4}\right),\end{cases}
$$

which cannot, in general, be solved in closed form. Analytic solutions are possible for $n=1$, given by (2.11), and for $n=0$ (constant compliance), where

$$
p(t)= \begin{cases}p_{\mathrm{b}} & \left(t_{1} \leq t \leq t_{2}\right) \\ p_{\mathrm{b}}+R Q_{\mathrm{inf}}-R Q_{\mathrm{inf}} \exp \left(-\frac{\tilde{e}}{R}\left(t-t_{2}\right)\right) & \left(t_{2}<t \leq t_{3}\right) \\ p_{\mathrm{b}}+\left(p\left(t_{3}^{-}\right)-p_{\mathrm{b}}\right) \exp \left(-\frac{e}{R}\left(t-t_{3}\right)\right) & \left(t_{3}<t \leq t_{4}\right)\end{cases}
$$

For other values of $n$, the ODE can only be solved numerically.

Wirth and Sobey (2008) also predicted that the reference pressure for the compliance should be

$$
p_{\mathrm{r}}=p_{\mathrm{ss}}+\Delta p-K,
$$




\section{A. EISENTRÄGER, I. SOBEY AND M. CZOSNYKA}

where $\Delta p$ is the pressure drop across the bridging veins and $K$ is a measure of the bending stiffness of the bridging veins. Following their discussion, the latter two terms are of similar size, so that the reference pressure $p_{\mathrm{r}}$ may be a rough approximation of the pressure in the sagittal sinus $p_{\mathrm{ss}}$. Hence, the production rate can be approximated, using this substitution in (2.6), as

$$
Q_{\text {prod }}=\frac{p_{\mathrm{b}}-p_{\mathrm{r}}}{R} .
$$

\section{Parameter Variations}

To obtain some intuition of the influences on ICP evolution of the different parameters, we consider variation of the parameters $E, R, p_{\mathrm{r}}, p_{\mathrm{b}}$ and $n$ in (2.17) around the average set of parameter values given in Table 1 For the case $n=$ 1 , the analytic solution (2.11) is used, otherwise ICP comes from a numerical solution of (2.17) from MATLAB's ode45. The results of these calculations, showing the intracranial pressure, inflow and outflow rate, and the increase in fluid volume are plotted in Figures 1-77 and discussed in detail below.

In each of the plots, a thick solid line represents the values obtained from the standard parameters and the values corresponding to the variations are plotted in various other line styles. Subfigure (a) shows the variations in CSF pressure (ICP) $p$. Both the inflow rates (grey) and the corresponding outflow rates (black) are given in subfigure (b), and the increase in CSF volume, integrated numerically from (2.13) is plotted in subfigure (c). In each figure, the legend of subfigure (a) applies to the other two plots as well.

Changes of the elastance $E$ have a linear effect on the slope of the pressure curve, thus scaling the curve horizontally. The plateau pressure is reached earlier for larger values of $E$ and later, or not at all during the given infusion time, for smaller values and similarly the baseline pressure is reached earlier or later after the infusion was stopped (Fig. 1(a)). The form of the outflow rate closely follows the form of the pressure curve (Fig. 1(b)), since the plateau pressure coincides with the outflow rate balancing the inflow rate. Consequently, the increase in fluid volume is higher for smaller values of $E$ and smaller for

TABLE 1: Standard parameter values.

\begin{tabular}{lrlrl}
\hline Start time of data & $t_{1}$ & $=0 \mathrm{~min}$ \\
Start time of infusion & $t_{2}$ & $=5 \mathrm{~min}$ \\
End time of infusion & $t_{3}$ & $=20 \mathrm{~min}$ \\
End time of data & $t_{3}$ & $=25 \mathrm{~min}$ \\
Infusion rate & $Q_{\text {inf }}$ & $=1.5 \mathrm{ml} \cdot(\mathrm{min})^{-1}$ \\
Elastance coefficient & $E$ & $=0.2$ & $(\mathrm{ml})^{-1}$ \\
Resistance & $R$ & $=15 \mathrm{mmHg} \cdot \mathrm{min} \cdot(\mathrm{ml})^{-1}$ \\
Reference pressure & $p_{\mathrm{r}}$ & $=5 \mathrm{mmHg}$ \\
Baseline pressure & $p_{\mathrm{b}}$ & $=10 \mathrm{mmHg}$ \\
\hline
\end{tabular}




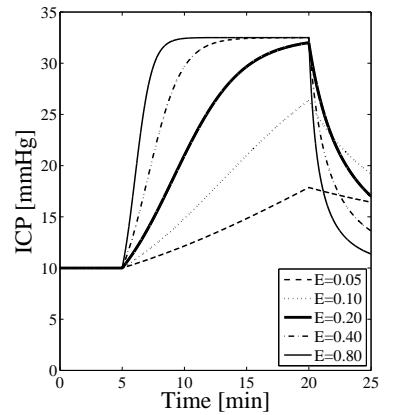

(a)

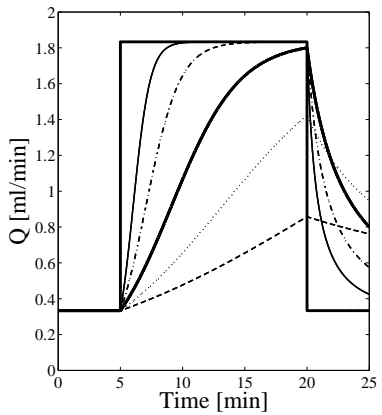

(b)

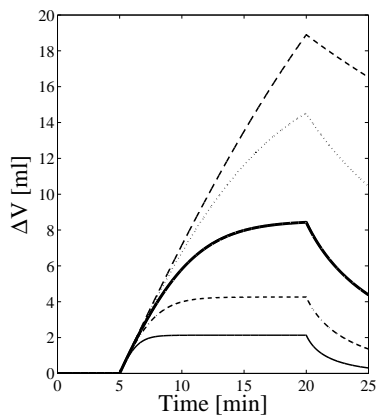

(c)

FIG. 1: Variation of $E$ in $\mathrm{ml}^{-1}$ : (a) intracranial pressure, (b) inflow (grey) and outflow (black), (c) increase in CSF volume.

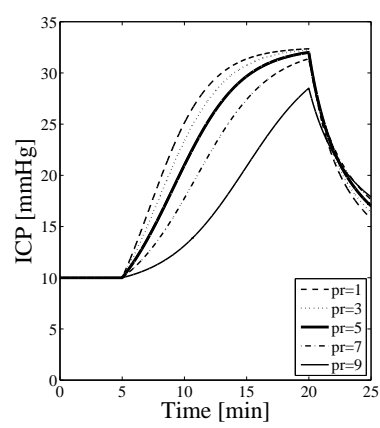

(a)

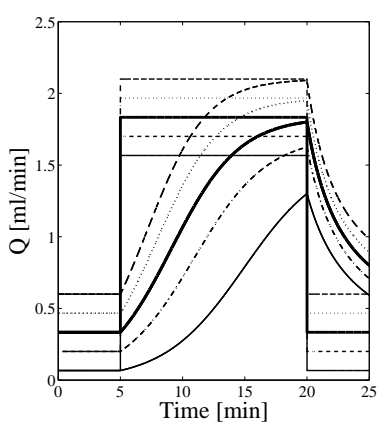

(b)

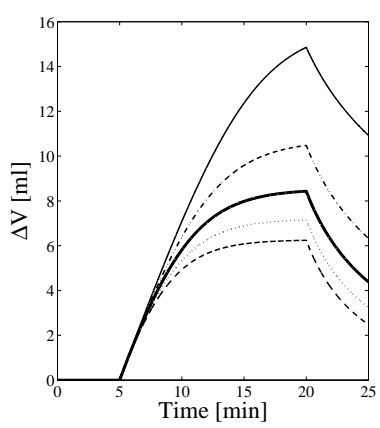

(c)

FIG. 2: Variation of $p_{\mathrm{r}}$ in mmHg: (a) intracranial pressure, (b) inflow (grey) and outflow (black), (c) increase in CSF volume.

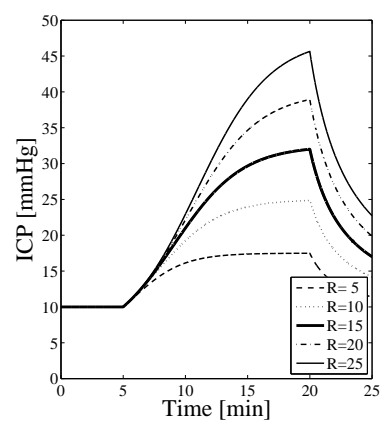

(a)

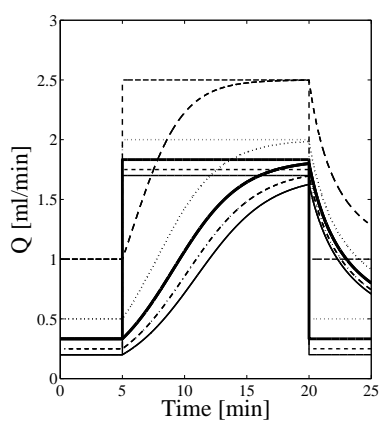

(b)

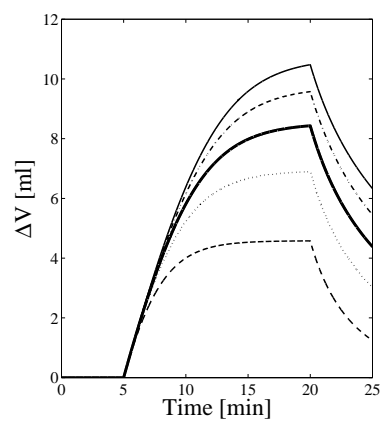

(c)

FIG. 3: Variation of $R$ in $\mathrm{mmHg} \cdot \mathrm{min} \cdot(\mathrm{ml})^{-1}$ : (a) intracranial pressure, (b) inflow (grey) and outflow (black), (c) increase in CSF volume. 


\section{A. EISENTRÄGER, I. SOBEY AND M. CZOSNYKA}

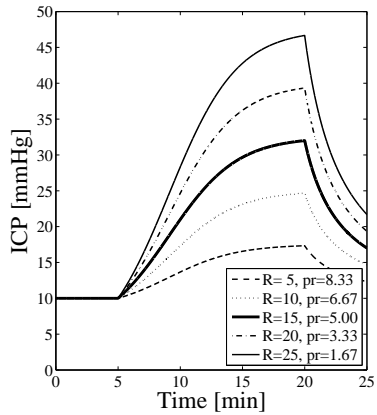

(a)

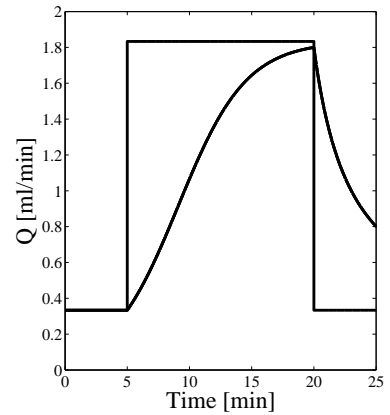

(b)

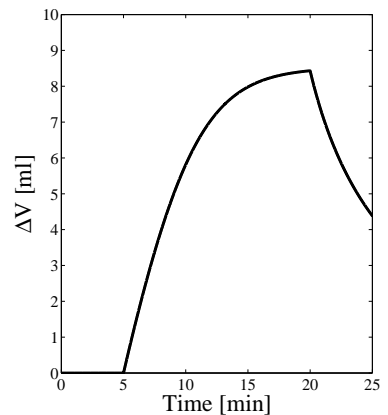

(c)

FIG. 4: Variation of $R$ and $p_{\mathrm{r}}$, with $Q_{\text {prod }}$ fixed: (a) intracranial pressure, (b) inflow (grey) and outflow (black), (c) increase in CSF volume.

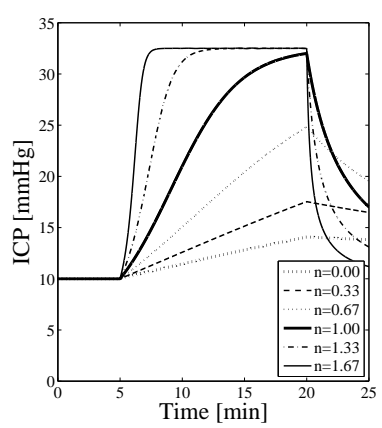

(a)

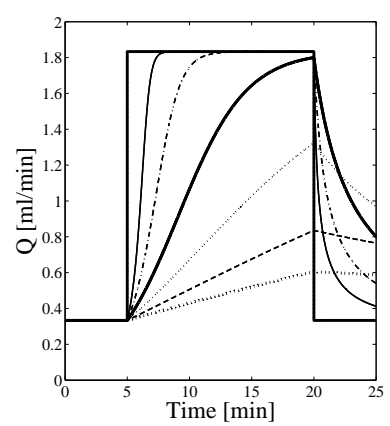

(b)

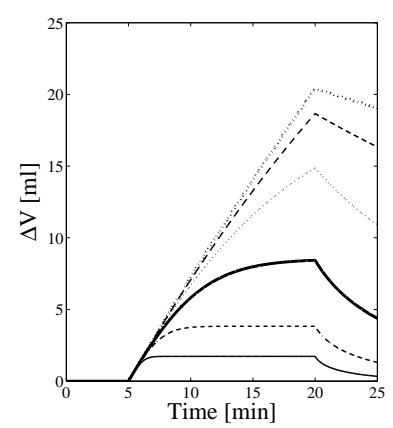

(c)

FIG. 5: Variation of $n$ : (a) intracranial pressure, (b) inflow (grey) and outflow (black), (c) increase in CSF volume.

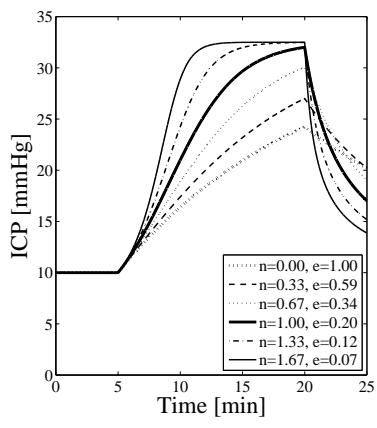

(a)

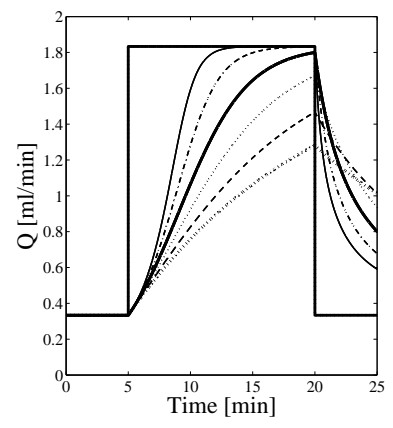

(b)

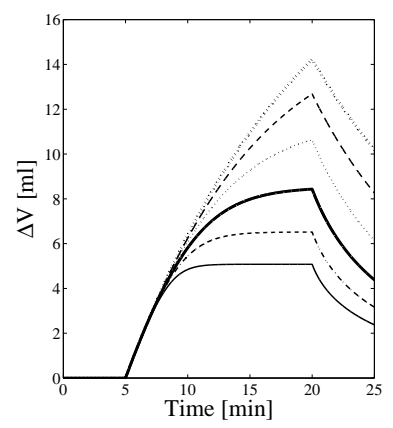

(c)

FIG. 6: Variation of $n$ and $\tilde{e}$, with $E\left(p_{\mathrm{b}}\right)$ fixed: (a) intracranial pressure, (b) inflow (grey) and outflow (black), (c) increase in CSF volume. 


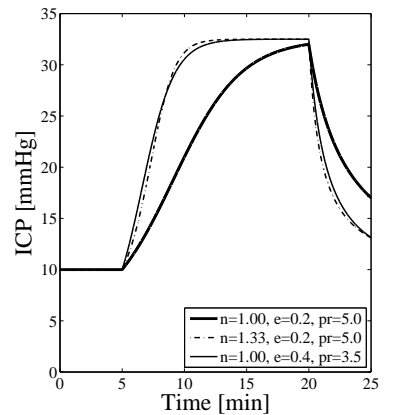

(a)

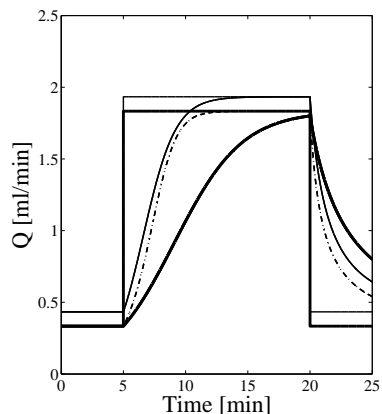

(b)

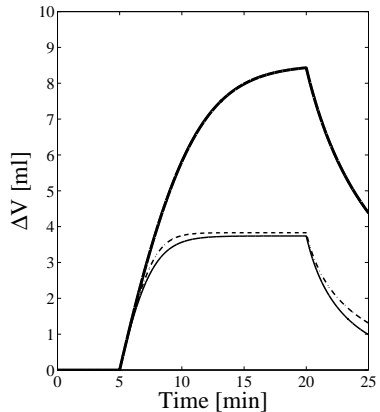

(c)

FIG. 7: Variation of $n, \tilde{e}$ and $p r$ : (a) intracranial pressure, (b) inflow (grey) and outflow (black), (c) increase in CSF volume.

larger values (Fig. 1(c)).

The actual form of the pressure curve between the baseline pressure and the plateau pressure can be changed by varying the reference pressure $p_{\mathrm{r}}$ (Fig. 2(a)). Lower values of this parameter correspond to a steepening of the pressure curve early after the change in inflow rate and a flattening thereafter. The outflow rate again follows the changes of the pressure curve, with a superimposed vertical shift of both, inflow and outflow rate, due to the change in CSF production rate (Fig. 2(b)). These changes result in a higher increase in CSF volume for higher reference pressure and vice versa (Fig. 2(c)). Adding the same value to both, the reference pressure $p_{\mathrm{r}}$ and the baseline pressure $p_{\mathrm{b}}$, only shifts the pressure curve up or down without any effect on its shape or on the flow and volume curves. A change in the baseline pressure only can be regarded as such a shift together with a change of the reference pressure.

The resistance $R$ is the only parameter that has an effect on the difference between the plateau and the baseline pressures (Fig. 3(a)), which is obvious from the volume balance in the new steady state $p_{\text {plateau }}-p_{\mathrm{b}}=R Q_{\text {inf }}$. For pressure values close to baseline pressure $p_{\mathrm{b}}$, the effect of the resistance on the pressure derivative diminishes, thus the slope of the pressure curve is similar at the beginning of the infusion. This results in the plateau pressure being reached earlier for smaller resistances, corresponding to smaller plateau pressures. Thus in these cases, the outflow and inflow rate balance earlier (Fig. 3(b)), and the increase in CSF volume is smaller (Fig. 3(c)). Since variations in the reference pressure or the resistance also change the CSF production rate, we next consider variations of both parameters together, with the production rate kept constant. In this case, the pressure curve between the baseline pressure and the plateau pressure is simply scaled to fit this range (Fig. $4(\mathrm{a})$ ), while there are no changes in the flow rates or fluid volume increase at all (Fig. $4(\mathrm{~b}),(\mathrm{c}))$. Hence the effects of variations in resistance can be viewed as a combination of this scaling together with a change in the reference pressure.

Considering the generalised model, variations of the power $n$ with constant 


\section{A. EISENTRÄGER, I. SOBEY AND M. CZOSNYKA}

$\tilde{e}$ have similar effects as changes in the elastance in the standard model (Fig. [5). This can be expected as the main influence of $n$ in this setting is on the size of the elastance $E(p)=\tilde{e}\left(p-p_{\mathrm{b}}\right)^{n-1}$. Only when keeping the value of $E$ constant at a specific pressure, for example for the baseline pressure, can we examine the influence of the power itself (Fig. 6). It is no surprise that the pressure curves look similar at the beginning of the infusion, since there, the pressure is still close to the baseline pressure and hence the elastance varies only slightly. Overall, increasing the power $n$ leads to a steepening of the pressure curve and the outflow rate, and thus to a smaller increase in CSF volume.

The example in Fig. 7 makes the nonlinear nature of the relationship between the parameters and the pressure curve even clearer. Two sets of different parameter values yield extremely similar values for the pressure curves, only a small shift in the flow curves and as with ICP, almost indistinguishable volume curves. These values were only chosen by hand. Using one set as a starting guess for a least squares fit to the ICP curve of the other set, would probably lead to even closer results. This nonlinearity makes the inverse problem of determining parameter values from observed ICP during an infusion test so hard to solve.

\section{Methodology}

\subsection{Data}

The entirely anonymous clinical data used in this study has come from infusion tests carried out at Addenbrooke's Hospital, Cambridge, UK. Each data set consists of the times when the infusion started and stopped, the constant infusion rate, CSF pressure, taken either from the ventricles or from the spinal column, and arterial blood pressure, usually measured via a finger cuff. The infusion lasts around 15 minutes, and both pressures are measured at a rate of 30 data points per second for the whole duration of the test, that is approximately ten minutes before until five minutes after the infusion, thus yielding about 54000 data points.

For most data sets, the beginning of the test $t_{1}$ coincides with the beginning of the measurements $t=0$, but in some cases, there are some jumps or drifts in the pressure before the infusion. This part of the data is not considered as these effects are most likely due to movements of the patient or a readjustment of the pressure sensor . Similar situations can occur after the infusion, for example a patient might sit up to relieve side effects such as headache and nausea. This kind of information is not available with the data sets, so the reliability of ICP measurement after the infusion is not known and in most cases, that data can not be used here. Hence, only the period between $t_{1}$ and $t_{3}$ is taken into account for parameter estimation and $\mathcal{T}$ is defined as the set containing measurement times in this acceptable range. 


\subsection{Pre- and Postprocessing}

Some straightforward preprocessing of the data will yield starting points for a least squares fit. To smooth out oscillations at heart rate, $p_{\mathrm{W}}(t)$ is defined as the mean of the measured CSF pressure $p_{\mathrm{m}}$ over a one minute window starting at time $t$ and $p_{\mathrm{w}}^{+}$and $p_{\mathrm{w}}^{-}$as the maximum and minimum value of $p_{\mathrm{w}}(t)$, respectively. With this, coarse guesses for the parameters can be defined as

$p_{\mathrm{b}}^{\mathrm{g}}=p_{\mathrm{w}}^{-}, \quad p_{\mathrm{r}}^{\mathrm{g}}=\max \left\{p_{\mathrm{b}}^{\mathrm{g}}-4 \mathrm{mmHg}, 1 \mathrm{mmHg}\right\}, \quad R^{\mathrm{g}}=\frac{p_{\mathrm{w}}^{+}-p_{\mathrm{b}}^{\mathrm{g}}}{Q_{\mathrm{inf}}}, \quad E^{\mathrm{g}}=0.25 \frac{1}{\mathrm{ml}}$.

The guess for the baseline pressure is taken as the minimal mean of the pressure, the reference pressure is assumed to lie $4 \mathrm{mmHg}$ below that, as the average values from Iuniewicz et al. (2005), $p_{\mathrm{b}}=10.7$ and $p_{\mathrm{r}}=6.6$, indicate. To force the latter to be positive, hence physical, it is set to $1 \mathrm{mmHg}$ if the value obtained is smaller than that. The assumption that the maximum of the average pressure is the new plateau pressure, that is a new steady state pressure for the increased inflow rate, yields the starting guess for $R$. The value for $E$ is again taken as a value typical for patients with hydrocephalus, similar to the mean value of $0.24 \mathrm{ml}^{-1}$ for 20 infusion tests from Juniewicz et al. (2005)

Having obtained the 'best' values for the parameters with one of the following fitting methods, an approximation of the production rate $Q_{\text {prod }}$ can be computed from (2.20) and the maximal increase in fluid volume $\Delta V$ can be estimated by numerically integrating (2.13).

\subsection{Parameter Estimation}

\subsubsection{Method A: Standard compliance model}

At first, the standard model in $\$ 2.2$ is considered, which is equivalent to setting $n=1$ in 2.3 . Letting the normalised unknown parameters be

$$
x_{1}=\frac{p_{\mathrm{b}}}{p_{\mathrm{b}}^{\mathrm{g}}}, \quad x_{2}=\frac{E}{R} \frac{R^{\mathrm{g}}}{E^{\mathrm{g}}}, \quad x_{3}=\frac{p_{\mathrm{r}}}{p_{\mathrm{r}}^{\mathrm{g}},} \quad x_{4}=\frac{R Q_{\mathrm{inf}}+p_{\mathrm{b}}}{R^{\mathrm{g}} Q_{\mathrm{inf}}^{\mathrm{g}}+p_{\mathrm{b}}^{\mathrm{g}}}
$$

the objective function

$$
F_{\mathrm{A}}: \mathbb{R}^{4} \mapsto \mathbb{R}: \quad x \mapsto \sum_{t \in \mathcal{T}}\left(p_{\mathrm{ana}}[x](t)-p_{\mathrm{m}}(t)\right)^{2}
$$

can be defined, where $p_{\text {ana }}[x]$ is given by (2.11) with the parameters

$$
p_{\mathrm{b}}=x_{1} p_{\mathrm{b}}^{\mathrm{g}}, \quad p_{\mathrm{r}}=x_{3} p_{\mathrm{r}}^{\mathrm{g}}, \quad R=\frac{1}{Q_{\mathrm{inf}}}\left(\left(R^{\mathrm{g}} Q_{\mathrm{inf}}^{\mathrm{g}}+p_{\mathrm{b}}^{\mathrm{g}}\right) x_{4}-p_{\mathrm{b}}\right), \quad E=x_{2} E^{\mathrm{g}} \frac{R}{R^{\mathrm{g}}} .
$$




\section{A. EISENTRÄGER, I. SOBEY AND M. CZOSNYKA}

The nonlinear least squares fit problem

$$
\min _{x \in \mathbb{R}^{4}} F_{\mathrm{A}}(x)
$$

is then solved using fminsearch in MATLAB with the starting value $x^{(0)}=$ $(1,1,1,1)$.

\subsubsection{Method B: Generalised compliance model with variable $n$ unknown}

In the second case, $n$ is allowed to vary as one of the parameters to be fitted. Defining furthermore

$$
n^{\mathrm{g}}=1, \quad \tilde{e}^{\mathrm{g}}=E^{\mathrm{g}}\left(p_{\mathrm{b}}^{\mathrm{g}}-p_{\mathrm{r}}^{\mathrm{g}}\right)^{\left(1-n^{\mathrm{g}}\right)}=E^{\mathrm{g}}
$$

and the normalised parameters

$$
x_{1}=\frac{p_{\mathrm{b}}}{p_{\mathrm{b}}^{\mathrm{g}}}, \quad x_{2}=\frac{\tilde{e}}{R} \frac{R^{\mathrm{g}}}{\tilde{e}^{\mathrm{g}}}, \quad x_{3}=\frac{p_{\mathrm{r}}}{p_{\mathrm{r}}^{\mathrm{g}}}, \quad x_{4}=\frac{R Q_{\mathrm{inf}}+p_{\mathrm{b}}}{R^{\mathrm{g}} Q_{\mathrm{inf}}^{\mathrm{g}}+p_{\mathrm{b}}^{\mathrm{g}}}, \quad x_{5}=n,
$$

the objective function is now a function of five variables

$$
F_{\mathrm{B}}: \mathbb{R}^{5} \mapsto \mathbb{R}: \quad x \mapsto \sum_{t \in \mathcal{T}}\left(p_{\text {num }}[x](t)-p_{\mathrm{m}}(t)\right)^{2},
$$

with the numerical solution $p_{\text {num }}[x]$ to the ODE (2.17) obtained via ode45 in MATLAB. Again, the minimisation is solved numerically with fminsearch.

\subsubsection{Method C: Generalised compliance model with $n$ predetermined}

The third case is very similar to method B, that is using the generalised model, but setting $n$ to a fixed given value $n^{\mathrm{g}} \neq 1$ suggested by other simulation results. This requires numerical solution of the governing differential equation within the optimisation routine but reduces the number of unknown parameters to be the same as in the standard model, thus

$$
\tilde{e}^{\mathrm{g}}=E^{\mathrm{g}}\left(p_{\mathrm{b}}^{\mathrm{g}}-p_{\mathrm{r}}^{\mathrm{g}}\right)^{\left(1-n^{\mathrm{g}}\right)} \neq E^{\mathrm{g}} .
$$

The guessed values are the same as in method B, that is either obtained from the results of method A or via the same preprocessing, and the same normalisation for parameters $x_{1}$ up to $x_{4}$ as in method $\mathrm{B}$ is used. The objective function is defined as

$$
F_{\mathrm{C}}: \mathbb{R}^{4} \mapsto \mathbb{R}: \quad x \mapsto F_{\mathrm{B}}\left(x_{1}, x_{2}, x_{3}, x_{4}, n^{\mathrm{g}}\right)
$$

and solved numerically with fminsearch. 
PARAMETER ESTIMATIONS FOR THE CSF INFUSION TEST

TABLE 2: Differences between artificial data sets.

\begin{tabular}{|c||c|c|c|c|}
\hline Set & AD1 & AD2 & AD3 & AD4 \\
\hline$n$ & 1 & 1 & 0.6 & 0.6 \\
\hline rand & no & yes & no & yes \\
\hline
\end{tabular}

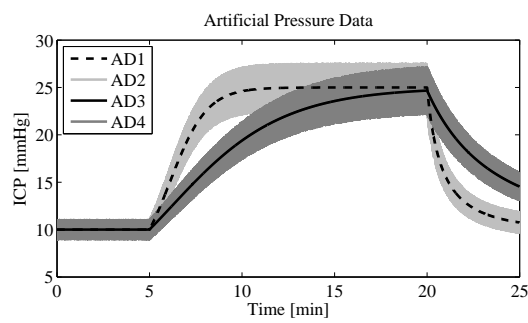

FIG. 8: Artificial Pressure Data: AD1 standard model, AD2 standard model with variance, $\mathrm{AD} 3$ generalised model, $\mathrm{AD} 4$ generalised model with variance.

\section{Tests with Artificial Data}

To test the models and implementation, four artificial data sets are created using known parameters and then parameter estimation carried out in these data sets and the results compared to the original known parameters. Two data sets are prepared for each model using $n=1$ and $n=0.6$, one set consisting of the pressure curve described by the models in section 2 , and another set where for each data point, the pressure at time $t$ was chosen randomly from the interval $[0.9 p(t), 1.1 \cdot p(t)]$ around the mean value with uniform distribution, to simulate unsteadiness in the data (see Table 2).

The common parameter values for all four artificial data sets shown in Fig. 8 are

$$
\tilde{e}=0.5 \frac{\mathrm{mmHg}^{(1-n)}}{\mathrm{ml}}, \quad R=10 \frac{\mathrm{mmHg} \mathrm{min}}{\mathrm{ml}}, \quad p_{\mathrm{b}}=10 \mathrm{mmHg}, \quad p_{\mathrm{r}}=5 \mathrm{mmHg} .
$$

The data sets AD1 and AD2 are used for methods A and B. Method C with exact $n$ would be equivalent to method A except for some small deviations due to the numerical integration of the ordinary differential equation (2.10) rather than using its analytic solution (2.11). Data sets AD3 and AD4 are used for all three methods of parameter estimations. The results of those tests can be found in Table 3. The relative errors in the parameter values are stated along with the relative error in the mean pressure, that is the fitted pressure compared to the pressure from AD1 for the two data sets created with the standard model, $\mathrm{AD} 1$ and $\mathrm{AD} 2$, the pressure from AD3 for the two data sets created with the generalised model, AD3 and AD4.

First of all, it can be observed that in all cases, both the baseline pressure $p_{\mathrm{b}}$ and the resistance to CSF outflow are always estimated accurately. This 


\section{A. EISENTRÄGER, I. SOBEY AND M. CZOSNYKA}

TABLE 3: Relative error norms for artificial data tests in the parameters values and the mean pressure.

\begin{tabular}{|c|c|c|c|c|c|c|c|}
\hline Set & $\mathrm{M}$ & $\left|\frac{n-n^{\mathrm{fit}}}{n}\right|$ & $\left|\frac{\tilde{\mathcal{e}}-\tilde{e}^{\mathrm{fit}}}{\tilde{e}}\right|$ & $\left|\frac{R-R^{\mathrm{fit}}}{R}\right|$ & $\left|\frac{p_{\mathrm{r}}-p_{\mathrm{r}}^{\mathrm{fit}}}{p_{\mathrm{r}}}\right|$ & $\frac{p_{\mathrm{b}}-p_{\mathrm{b}}^{\mathrm{fit}}}{p_{\mathrm{b}}}$ & $\left\|\frac{p-p^{\mathrm{fit}}}{p}\right\|_{\infty}$ \\
\hline \hline AD1 & A & - & $6 \cdot 10^{-4}$ & $4 \cdot 10^{-6}$ & $2 \cdot 10^{-3}$ & $1 \cdot 10^{-5}$ & $2 \cdot 10^{-4}$ \\
AD1 & $\mathrm{B}$ & 0.16 & 0.42 & $3 \cdot 10^{-5}$ & 0.32 & $3 \cdot 10^{-4}$ & $2 \cdot 10^{-3}$ \\
\hline AD2 & A & - & $2 \cdot 10^{-2}$ & $1 \cdot 10^{-3}$ & $2 \cdot 10^{-2}$ & $4 \cdot 10^{-4}$ & $1 \cdot 10^{-3}$ \\
AD2 & B & 0.14 & 0.39 & $1 \cdot 10^{-3}$ & 0.31 & $6 \cdot 10^{-4}$ & $2 \cdot 10^{-3}$ \\
\hline AD3 & A & 0.67 & 0.75 & $4 \cdot 10^{-3}$ & 1.28 & $5 \cdot 10^{-4}$ & $3 \cdot 10^{-3}$ \\
AD3 & B & 0.21 & 0.34 & $1 \cdot 10^{-3}$ & 0.39 & $2 \cdot 10^{-4}$ & $1 \cdot 10^{-3}$ \\
AD3 & C & - & $4 \cdot 10^{-4}$ & $7 \cdot 10^{-6}$ & $1 \cdot 10^{-3}$ & $1 \cdot 10^{-5}$ & $1 \cdot 10^{-4}$ \\
\hline AD4 & A & 0.67 & 0.75 & $7 \cdot 10^{-3}$ & 1.21 & $4 \cdot 10^{-5}$ & $3 \cdot 10^{-3}$ \\
AD4 & B & 0.24 & 0.37 & $5 \cdot 10^{-3}$ & 0.40 & $3 \cdot 10^{-4}$ & $1 \cdot 10^{-3}$ \\
AD4 & C & - & $2 \cdot 10^{-2}$ & $3 \cdot 10^{-3}$ & $4 \cdot 10^{-2}$ & $6 \cdot 10^{-4}$ & $1 \cdot 10^{-3}$ \\
\hline
\end{tabular}

is no surprise, as the baseline pressure equals the mean of the pressure before the infusion and together, these two parameters define the plateau value of the pressure during the infusion. Furthermore, the mean ICP pressure of the estimated parameters fits the model values. A relative error of the order of $10^{-3}$ is well within the expected error margin, taking into account that the measurements are hardly as/more accurate. Nevertheless, in most cases huge differences are obtained between the estimated and the real values of the other model parameters.

Method A obtained reasonable results for all parameters for the data sets $\mathrm{AD} 1$ and AD2. This is reassuring that the least squares implementation is principally correct, as here the standard model is fitted to itself. Only the variation of the pressure values in set AD2 increase the errors to some extent, but still keeping them within very few per cent. However, if the power $n$ is allowed to vary as well, by using method $B$, which contains the standard model and thus the correct solution as a special case, the estimates for $n, \tilde{e}, p_{\mathrm{r}}$, and thus $E\left(p_{\mathrm{b}}\right)$, are far from the correct values. The objective function for this problem seems to have become too ill conditioned, containing several local minima.

Applying least squares fit method A to data sets AD3 and AD4 must necessarily lead to incorrect parameter estimates, as $n$ is set to the incorrect value one rather than 0.6. The relative errors for $n, \tilde{e}, p_{\mathrm{r}}$ and $E\left(p_{\mathrm{b}}\right)$ are all of the order of one, so the results in these cases are completely unreliable. On the other hand, the pressure is fitted similarly well. This means that a good fit for the pressure can be obtain with the standard model even though this model might not really describe what is going on. Method B suffers again from the ill conditioning and the likely existence of several local minima of the objective function. Hence, reasonable estimates for parameters appears to be obtained only if the value for $n$ is known in advance and fixed as is the case for method A and C here. 
PARAMETER ESTIMATIONS FOR THE CSF INFUSION TEST

TABLE 4: Clinical data for infusion test sets: Start of test, start of infusion, end of infusion, end of test, infusion rate, mean arterial blood pressure.

\begin{tabular}{|c|c|c|c|c|c|c|}
\hline \multirow{2}{*}{ Set } & \multicolumn{4}{|c|}{ Times [min] } & \multirow{2}{*}{$\begin{array}{c}Q_{\text {inf }} \\
{[\mathrm{ml} / \mathrm{min}]}\end{array}$} & \multirow{2}{*}{$\begin{array}{c}\mathrm{ABP} \\
{[\mathrm{mmHg}]}\end{array}$} \\
\hline & $t_{1}$ & $t_{2}$ & $t_{3}$ & $t_{4}$ & & \\
\hline 00 & $\overline{0.0}$ & $\overline{5.5}$ & 19.9 & $\overline{24.0}$ & $\overline{c 1.5}$ & 86 \\
\hline 01 & 0.0 & 6.7 & 13.7 & 26.7 & 1.5 & 107 \\
\hline 02 & 0.0 & 5.4 & 34.4 & 36.3 & 1.5 & 68 \\
\hline 03 & 9.0 & 15.3 & 34.8 & 38.7 & 1.5 & 52 \\
\hline 04 & 0.0 & 8.3 & 24.8 & 31.8 & 1.5 & 67 \\
\hline 05 & 0.0 & 8.0 & 19.0 & 27.5 & 1.5 & 70 \\
\hline 06 & 12.0 & 14.8 & 39.8 & 47.1 & 1.5 & 93 \\
\hline 07 & 0.0 & 12.7 & 26.7 & 27.0 & 1.5 & 125 \\
\hline 08 & 0.0 & 13.0 & 27.0 & 31.7 & 1.5 & 68 \\
\hline 09 & 0.0 & 4.0 & 13.0 & 23.9 & 1.0 & 85 \\
\hline 10 & 0.0 & 4.5 & 20.5 & 26.0 & 1.5 & 57 \\
\hline
\end{tabular}

\section{Application to Clinical Data}

Timing and infusion rates for eleven clinical data sets are given in Table 4 This provides for each data set the beginning and end time of the test, $t_{1}$ and $t_{4}$, and of the infusion, $t_{2}$ and $t_{3}$, as well as the infusion rate $Q_{\text {inf }}$ and the overall mean arterial blood pressure. The results of the least squares fits for those data sets with the three different methods are given in Table 5 and the corresponding plots can be found in Figures $9+19$. For each data set, the measured ICP (light grey) is plotted together with the pressure curves obtained from the least squares fits (darker lines) into subfigure (a). In subfigure (b), the difference between the outflow rate for each least squares fit, obtained by inserting the measured ICP into equation (2.3), and the production rate is given (dark lines), as well as the infusion rate (light grey). Numerical integration of (2.13) using the outflow rate from subfigure (b) yields the increase in CSF volume plotted in subfigure (c). The legend of subfigure (c) similarly applies to subfigures (a) and (b).

Results where the reference pressure was greater than the baseline pressure or the measured pressure are not stated, because this leads to complex pressure values, which are obviously unphysical. The idea to use constrained optimisation to ensure physically reasonable parameter values was abandoned due to the uncertainty of where physiologically reasonable values end, the highly increased computational time of up to several weeks for each data set for tests with only physical constraints and several of the resulting parameters lying on the constraints in many of these cases, such as $p_{\mathrm{b}}=p_{\mathrm{r}}=0$, which again leads to unphysical or unphysiological results. 


\section{A. EISENTRÄGER, I. SOBEY AND M. CZOSNYKA}

The first column to look at should be the last, stating

$$
L:=\log _{10}\left(\frac{F_{B}(x)}{|\mathcal{T}|}\right)
$$

the base 10 logarithm of the objective function value for the resulting parameters normalised by the number of data points as a measure for the comparative quality of the different least squares solutions of the same data set. The objective function $F_{B}$, comparing the numerical solution for the pressure with the measurements, is used here for all three methods to make the values comparable. Although in method A, the analytic solution is used in the minimisation, this is a sufficiently close approximation of the objective function. For most of the data sets, the results from method B, that is keeping the power $n$ variable as well, are indeed the 'best' solution. However, the differences in $L$ between the models are so small that, given the finite tolerance of the numerical methods, the finite measurement accuracy, and particularly the inherent oscillations of the pressure, for any data set, all the obtained parameter sets given in Table 5 can be considered equally well fitted to the data.

As for the tests with artificial data sets in section 5 , the baseline pressure is obtained consistently by all three methods. The same holds for the resistance to outflow $R$, except for the data sets 01 and 09. In these two cases, the infusion had to be stopped before the plateau pressure was clearly reached (Fig. 10. 18), as higher intracranial pressures would have endangered the patient. Thus for these data sets, it is not possible to reliably extract the plateau pressure and hence the resistance or any of the other parameters from the measured pressure data.

Many of the other data sets inhibit similar problems of the pressure data not really matching the form presented by the model, but the effects are not always as obvious. For example in data sets 05 and 10, the plateau pressure has not properly been reached either. This is clearest from the plots of the volume increase (Fig 14(c), 19(c)), which should also approach a new plateau value. Here, it seems that the value for the resistance has been obtained correctly, but in both cases the values for the elastance and the reference pressure obtained with either method look highly unphysiological or unphysical, respectively. However, it is is not clear whether these observations are related. Furthermore, many pressure plots show waves on times scales of a few minutes. These waves could not yet be explained biologically or physically, hence, they cannot be modelled mathematically. Data set 08 (Fig. 17) is a perfect example. In a least squares algorithm, as was used here, some large deviations from the mean have a much bigger effect than several small ones, thus potentially leading to an incorrect estimate of the plateau pressure. For data set 08 , the plateau pressure is likely to be overestimated, yielding an underestimation of the resistance and further errors in the other parameter values.

Even if the quality of the data is good, such as in the data sets 00 and 02 (Fig. 9, 11), the estimates for $n, \tilde{e}$ and $p_{\mathrm{r}}$, or alternatively $E$ and $p_{\mathrm{r}}$, are not very reliable. This is due to the fact that these parameters enter the model indirectly 
PARAMETER ESTIMATIONS FOR THE CSF INFUSION TEST

TABLE 5: Results of Parameter Estimations: $n, \tilde{e}$ in $\mathrm{mmHg}^{(1-n)} / \mathrm{ml}, E\left(p_{\mathrm{b}}\right)$ in $\mathrm{ml}^{-1}, R$ in $\mathrm{mmHg} \cdot \mathrm{min} / \mathrm{ml}, p_{\mathrm{r}}$ and $p_{\mathrm{b}}$ in $\mathrm{mmHg}, Q_{\text {prod }}$ in $\mathrm{ml} / \mathrm{min}, \Delta V$ in $\mathrm{ml}$, measure for the quality of the fit $L$.

\begin{tabular}{|c|c|c|c|c|c|c|c|c|c|c|}
\hline Set & $\mathrm{M}$ & $n$ & $\tilde{e}$ & $E\left(p_{\mathrm{b}}\right)$ & $R$ & $p_{\mathrm{r}}$ & $p_{\mathrm{b}}$ & $Q_{\text {prod }}$ & $\Delta V\left(t_{3}\right)$ & $L$ \\
\hline 00 & $\bar{A}$ & $\overline{|c| 00}$ & $\overline{0.24}$ & 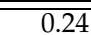 & 15.43 & 2.13 & 99.97 & 0.51 & 5.65 & 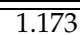 \\
\hline 00 & B & 3.08 & $2 \cdot 10^{-5}$ & 0.05 & 15.44 & -30.26 & 9.83 & 2.60 & 5.48 & 1.172 \\
\hline 00 & C & 0.50 & 1.25 & 0.91 & 15.64 & 8.06 & 9.95 & 0.12 & 5.83 & 1.175 \\
\hline 01 & A & 1.00 & 0.17 & 0.17 & 455.45 & 7.95 & 14.83 & 0.02 & 10.32 & 1.029 \\
\hline 01 & B & 0.70 & 0.56 & 0.47 & 92.04 & 13.03 & 14.88 & 0.02 & 9.64 & 1.028 \\
\hline 01 & C & 0.75 & 0.40 & 0.30 & $2 \cdot 10^{+3}$ & 11.37 & 14.85 & 0.01 & 10.46 & 1.028 \\
\hline 02 & $\mathrm{~A}$ & 1.00 & 0.16 & 0.16 & 4.23 & 5.70 & 6.17 & 0.11 & 17.02 & -0.121 \\
\hline 02 & B & 6.30 & $1 \cdot 10^{-8}$ & 0.01 & 4.20 & -6.77 & 6.09 & 3.06 & 16.20 & -0.114 \\
\hline 02 & C & 0.75 & 0.23 & 0.40 & 4.25 & 6.08 & 6.20 & 0.03 & 17.38 & -0.123 \\
\hline 03 & A & 1.00 & 0.36 & 0.36 & 10.18 & 4.69 & 7.75 & 0.30 & 4.96 & 1.361 \\
\hline 03 & B & 0.55 & 1.30 & 2.61 & 10.19 & 7.58 & 7.79 & 0.02 & 5.04 & 1.360 \\
\hline 03 & C & 0.50 & 1.44 & 5.08 & 10.18 & 7.71 & 7.79 & $8 \cdot 10^{-3}$ & 5.06 & 1.360 \\
\hline 04 & $\mathrm{~A}$ & 1.00 & 0.53 & 0.53 & 5.15 & 10.72 & 11.47 & 0.14 & 4.62 & 0.775 \\
\hline 04 & B & 5.03 & $1 \cdot 10^{-6}$ & 0.04 & 5.17 & -2.62 & 11.45 & 2.72 & 4.61 & 0.773 \\
\hline 04 & C & 0.75 & 0.87 & 1.30 & 5.15 & 11.27 & 11.48 & 0.04 & 4.62 & 0.775 \\
\hline 05 & $\mathrm{~A}$ & 1.00 & $5 \cdot 10^{-6}$ & $5 \cdot 10^{-6}$ & 8.66 & $-2 \cdot 10^{+5}$ & 11.46 & $2 \cdot 10^{+4}$ & 8.82 & 0.789 \\
\hline 05 & B & 0.22 & 0.34 & $4 \cdot 10^{-3}$ & $\begin{array}{l}8.00 \\
8.64\end{array}$ & -248.64 & 11.46 & 30.12 & $\begin{array}{l}.02 \\
8.80\end{array}$ & 0.789 \\
\hline 05 & C & 0.10 & $\begin{array}{l}0.04 \\
0.28\end{array}$ & $1 \cdot 10^{-6}$ & $\begin{array}{l}8.04 \\
8.67\end{array}$ & $-1 \cdot 10^{+6}$ & 11.46 & $1 \cdot 10^{+5}$ & $\begin{array}{l}8.00 \\
8.82\end{array}$ & 0.789 \\
\hline 05 & C & 0.30 & 0.02 & $2 \cdot 10^{-6}$ & 8.66 & $-6 \cdot 10^{+5}$ & 11.46 & $7 \cdot 10^{+4}$ & 8.82 & 0.789 \\
\hline 05 & C & 0.50 & $2 \cdot 10^{-3}$ & $2 \cdot 10^{-6}$ & 8.66 & $-5 \cdot 10^{+5}$ & 11.46 & $6 \cdot 10^{+4}$ & 8.82 & 0.789 \\
\hline 05 & C & 0.75 & $4 \cdot 10^{-5}$ & $1 \cdot 10^{-6}$ & 8.66 & $-9 \cdot 10^{+5}$ & 11.46 & $1 \cdot 10^{+5}$ & 8.82 & 0.789 \\
\hline 06 & $\mathrm{~A}$ & 1.00 & 0.12 & 0.12 & 12.44 & 2.78 & 7.56 & 0.38 & 13.24 & 0.998 \\
\hline 06 & B & 2.40 & $6 \cdot 10^{-4}$ & 0.03 & 12.23 & -10.81 & 7.63 & 1.15 & 12.97 & 0.997 \\
\hline 06 & C & 0.50 & 0.52 & 0.67 & 12.65 & 6.99 & 7.60 & 0.05 & 13.73 & 1.000 \\
\hline 06 & C & 0.75 & 0.26 & 0.21 & 12.52 & 5.01 & 7.57 & 0.20 & 13.41 & 0.999 \\
\hline 07 & A & 1.00 & 0.48 & 0.48 & 10.97 & 7.86 & 8.43 & 0.05 & $\begin{array}{l}7.12 \\
\end{array}$ & 0.941 \\
\hline 07 & B & 4.81 & $2 \cdot 10^{-6}$ & 0.04 & 10.70 & -5.36 & 8.45 & 1.29 & 6.81 & 0.934 \\
\hline 07 & C & 0.75 & 0.80 & 1.44 & 11.11 & 8.33 & 8.43 & $9 \cdot 10^{+3}$ & 7.30 & 0.943 \\
\hline 08 & $\mathrm{~A}$ & 1.00 & 0.08 & 0.08 & 11.76 & -12.93 & 8.05 & 1.78 & 7.52 & 1.264 \\
\hline 08 & B & 4.72 & $2 \cdot 10^{-9}$ & 0.02 & 11.39 & -70.33 & 8.08 & 6.88 & 7.14 & 1.263 \\
\hline 08 & C & 0.50 & 0.49 & 0.14 & 11.97 & -4.24 & 8.04 & 1.02 & 7.72 & 1.264 \\
\hline 09 & $\mathrm{~A}$ & 1.00 & 0.38 & 0.38 & 47.61 & 19.12 & 20.39 & 0.03 & 7.64 & 0.395 \\
\hline 09 & B & 0.58 & 1.02 & 11.94 & 79.41 & 20.53 & 20.54 & $4 \cdot 10^{-5}$ & 8.21 & 0.386 \\
\hline 09 & C & 0.75 & 0.67 & 0.85 & 72.32 & 20.03 & 20.44 & $6 \cdot 10^{-3}$ & 8.11 & 0.390 \\
\hline 10 & $\mathrm{~A}$ & 1.00 & $9 \cdot 10^{-7}$ & $9 \cdot 10^{-7}$ & 14.15 & $-1 \cdot 10^{+6}$ & 12.59 & $1 \cdot 10^{+3}$ & 12.73 & 0.983 \\
\hline 10 & C & 0.50 & $3 \cdot 10^{-4}$ & $5 \cdot 10^{-8}$ & 14.15 & $-2 \cdot 10^{+7}$ & 12.59 & $2 \cdot 10^{+6}$ & 12.73 & 0.983 \\
\hline
\end{tabular}




\section{A. EISENTRÄGER, I. SOBEY AND M. CZOSNYKA}

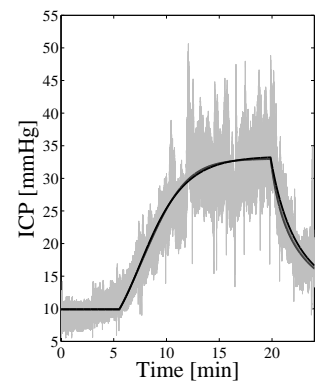

(a)

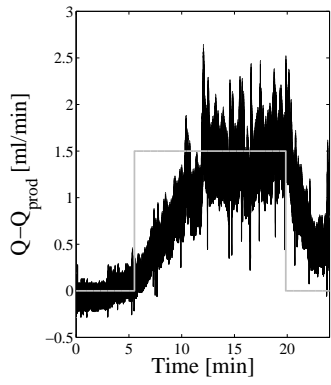

(b)

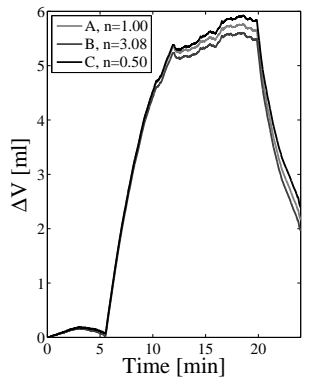

(c)

FIG. 9: Data Set 00: (a) measured intracranial pressure (light grey, thin line) and model intracranial pressures (darker, thick lines), (b) infusion rate (light grey, thick line) and increase in outflow rate (darker, thin lines), (c) CSF volume increase.

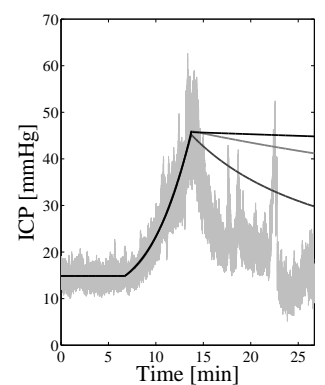

(a)

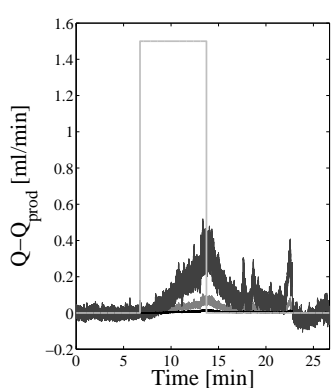

(b)

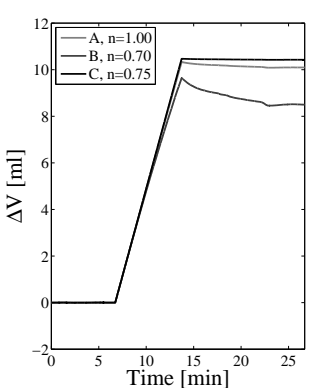

(c)

FIG. 10: Data Set 01: (a) measured intracranial pressure (light grey, thin line) and model intracranial pressures (darker, thick lines), (b) infusion rate (light grey, thick line) and increase in outflow rate (darker, thin lines), (c) CSF volume increase.

via the compliance $C(p)$. Only the form of the compliance function for a certain pressure range is of importance and that can be very similar for different sets of the parameters. The results of these interrelations can be seen most clearly in the estimates obtained with method B. Big values for $n$, small values for $\tilde{e}$ and large values for $p_{\mathrm{b}}-p_{\mathrm{r}}$ always occur together.

The estimate for the production rate $Q_{\text {prod }}$ is computed as part of the postprocessing from $p_{\mathrm{b}}, p_{\mathrm{ss}}=p_{\mathrm{r}}$ and $R$ via (2.20) and obviously suffers from poor results for those parameters. Hence, it is not surprising that many values are far off from the normal value of $0.35 \mathrm{ml} / \mathrm{min}$ cited in the literature (Nolte, 2009). However, in the estimate for the increase in CSF volume $\Delta V$ as in (2.13), the production rate is cancelled out so that consistent values are obtained again. 


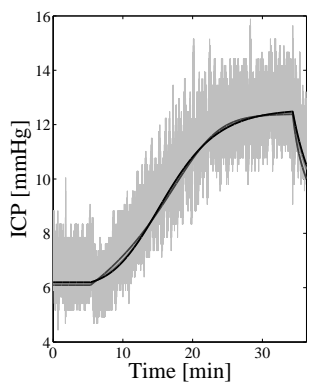

(a)

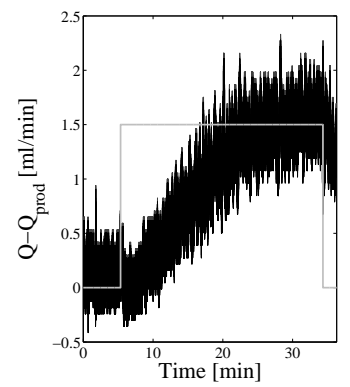

(b)

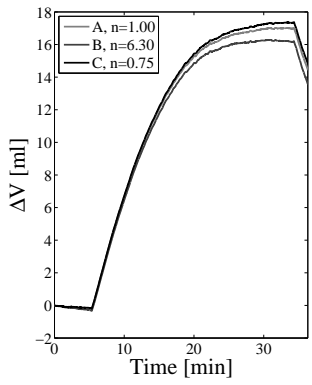

(c)

FIG. 11: Data Set 02: (a) measured intracranial pressure (light grey, thin line) and model intracranial pressures (darker, thick lines), (b) infusion rate (light grey, thick line) and increase in outflow rate (darker, thin lines), (c) CSF volume increase.

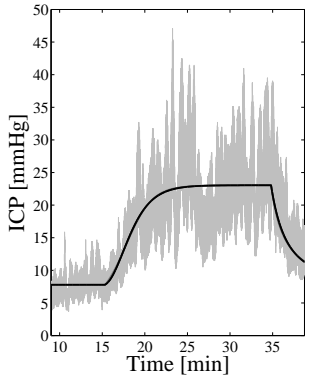

(a)

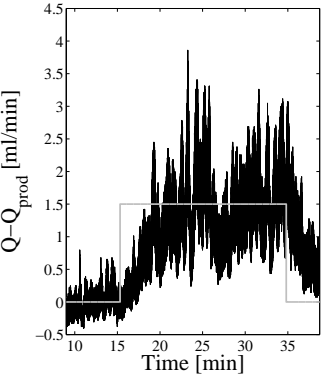

(b)

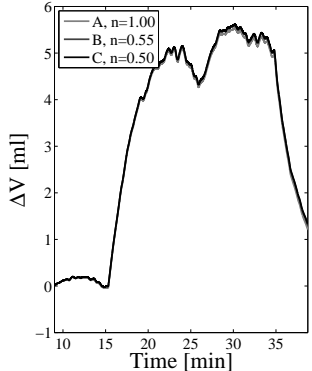

(c)

FIG. 12: Data Set 03: (a) measured intracranial pressure (light grey, thin line) and model intracranial pressures (darker, thick lines), (b) infusion rate (light grey, thick line) and increase in outflow rate (darker, thin lines), (c) CSF volume increase.

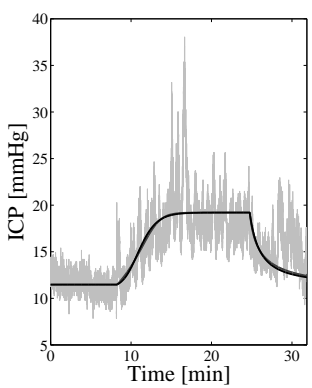

(a)

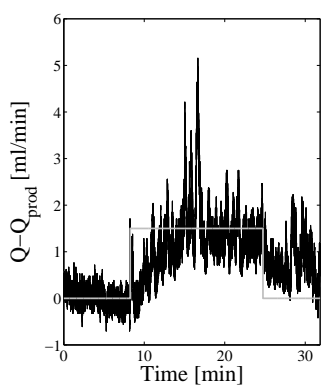

(b)

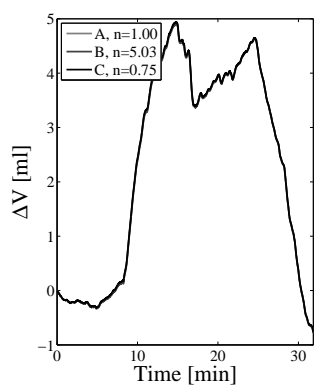

(c)

FIG. 13: Data Set 04: (a) measured intracranial pressure (light grey, thin line) and model intracranial pressures (darker, thick lines), (b) infusion rate (light grey, thick line) and increase in outflow rate (darker, thin lines), (c) CSF volume increase. 


\section{A. EISENTRÄGER, I. SOBEY AND M. CZOSNYKA}

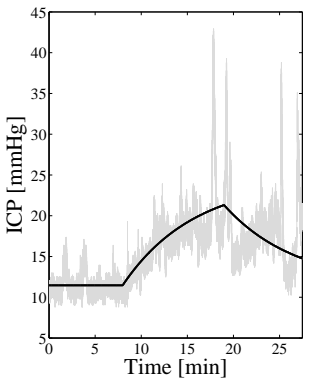

(a)

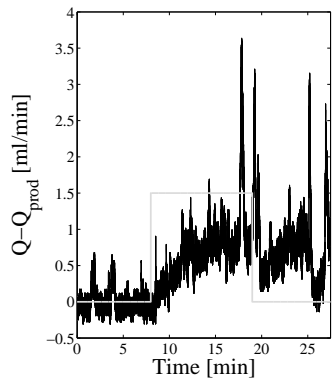

(b)

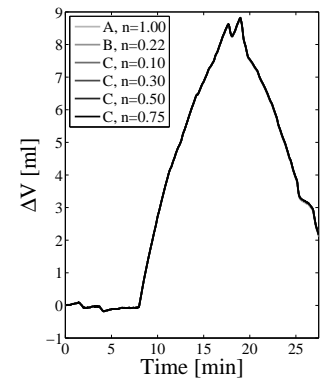

(c)

FIG. 14: Data Set 05: (a) measured intracranial pressure (light grey, thin line) and model intracranial pressures (darker, thick lines), (b) infusion rate (light grey, thick line) and increase in outflow rate (darker, thin lines), (c) CSF volume increase.

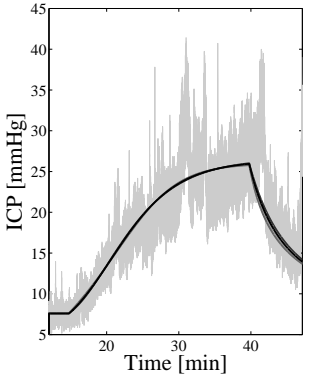

(a)

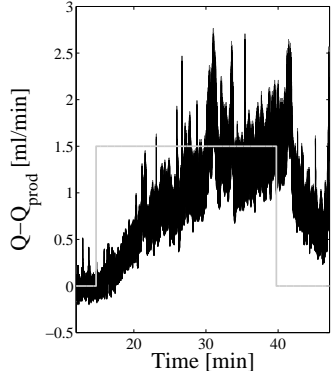

(b)

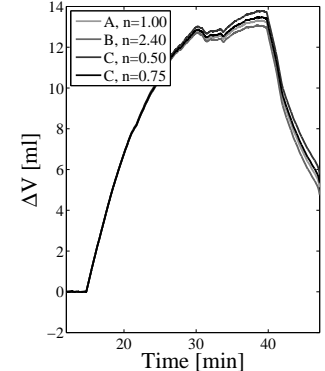

(c)

FIG. 15: Data Set 06: (a) measured intracranial pressure (light grey, thin line) and model intracranial pressures (darker, thick lines), (b) infusion rate (light grey, thick line) and increase in outflow rate (darker, thin lines), (c) CSF volume increase.

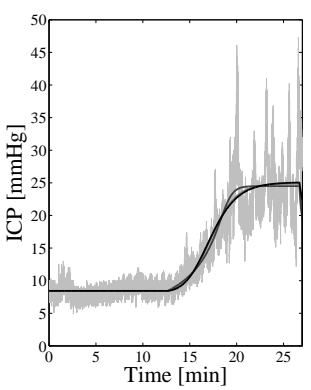

(a)

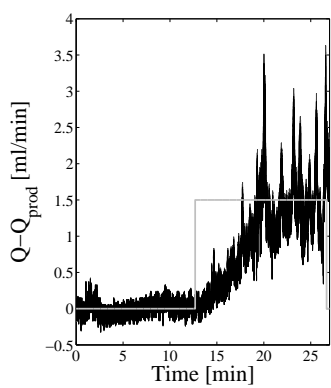

(b)

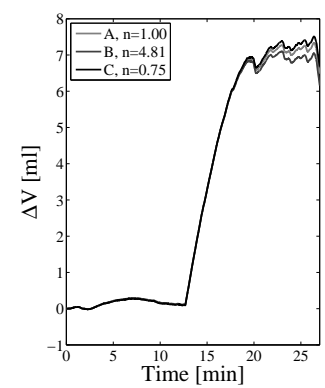

(c)

FIG. 16: Data Set 07: (a) measured intracranial pressure (light grey, thin line) and model intracranial pressures (darker, thick lines), (b) infusion rate (light grey, thick line) and increase in outflow rate (darker, thin lines), (c) CSF volume increase. 


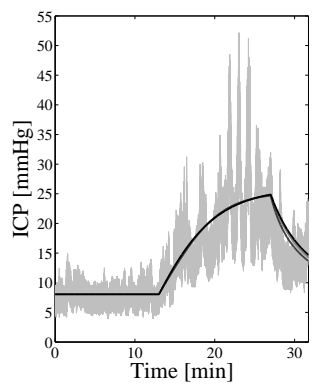

(a)

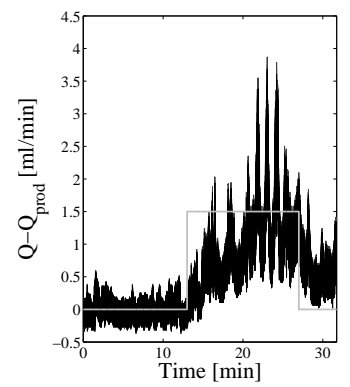

(b)

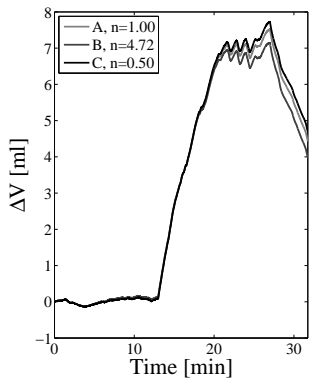

(c)

FIG. 17: Data Set 08: (a) measured intracranial pressure (light grey, thin line) and model intracranial pressures (darker, thick lines), (b) infusion rate (light grey, thick line) and increase in outflow rate (darker, thin lines), (c) CSF volume increase.

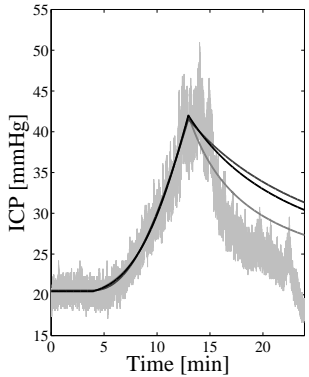

(a)

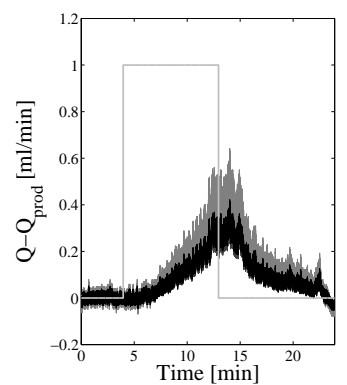

(b)

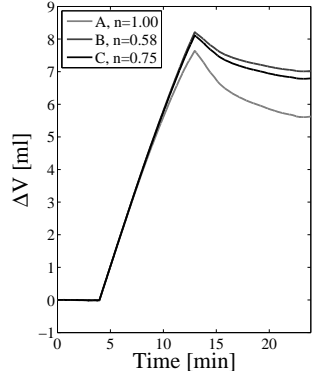

(c)

FIG. 18: Data Set 09: (a) measured intracranial pressure (light grey, thin line) and model intracranial pressures (darker, thick lines), (b) infusion rate (light grey, thick line) and increase in outflow rate (darker, thin lines), (c) CSF volume increase.

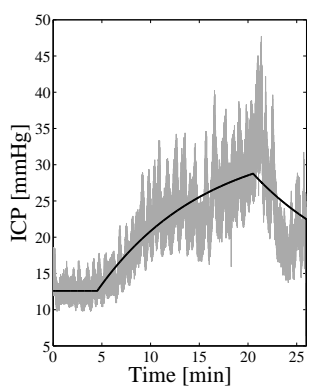

(a)

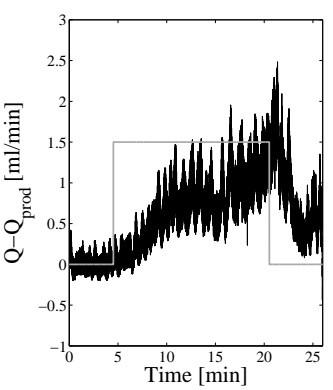

(b)

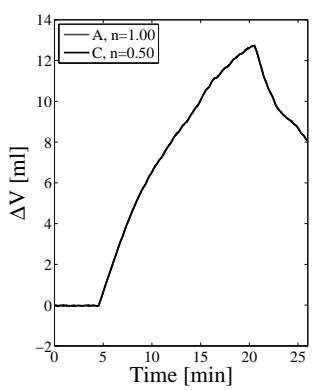

(c)

FIG. 19: Data Set 10: (a) measured intracranial pressure (light grey, thin line) and model intracranial pressures (darker, thick lines), (b) infusion rate (light grey, thick line) and increase in outflow rate (darker, thin lines), (c) CSF volume increase. 


\section{A. EISENTRÄGER, I. SOBEY AND M. CZOSNYKA}

\section{Conclusions}

It is clear from the results that not all parameters can be estimated reliably from an infusion test using the least squares fit described above. As is presently used in clinical assessment, the resistance, the baseline pressure and the increase in CSF volume can be extracted most reliably, whereas for the generalised compliance model, the additional parameter caused problems and hence attempting to use ICP observations to refine details of CSF compliance is not possible.

Given the uncertainty of what reference pressure should be used, it is possible that more refined testing, for example using different CSF infusion rates during one test and looking at the compliance over a reasonable pressure range might give more insight into the underlying CSF compliance and associated parameters.

It will also be the case that the quality of the parameter estimates depends on the quality of the data and the presence of temporal waves in the data or an early termination of the infusion will deteriorate the reliability of the results. The Euclidean norm is particularly sensitive to large deviations from the mean. So in cases where there are large amplitude waves or CSF oscillations, slightly better results might be obtained when using the $L_{1}$ norm, that is, substituting absolute values of the pressure differences for their squares in the objective function of the minimisation problem, but the underlying difficulty that parameter estimation from single compartment CSF modelling with different compliance functions is ill posed will remain.

\section{References}

Andersson, N., Malm, J., and Eklund, A. (2008). Dependency of cerebrospinal fluid outflow resistance om intracranial pressure. J. Neurosurg, 109, 918-922.

Czosnyka, M., Czosnyka, Z., Momjian, S., and Pickard, J. (2004). Cerebrospinal fluid dynamics. Physiological Measurement, 25, R51-R76.

Juniewicz, H., Kasprowicz, M., Czosnyka, M., Czosnyka, Z., Gizewski, S., Dzik, M., and Pickard, J. D. (2005). Analysis of intracranial pressure during and after the infusion test in patients with communicating hydrocephalus. Physiological Measurement, 26, 1039-1048.

Marmarou, A., Shulman, K., and Rosende, R. M. (1978). A nonlinear analysis of the cerebrospinal fluid system and intracranial pressure dynamics. J Neurosurg, 48(3), 332-344.

Nolte, J. (2009). The Human Brain: An Introduction to its Functional Anatomy. Mosby Elsevier, sixth edition edition.

Wirth, B. and Sobey, I. (2008). A model for an inverse power constitutive law for cerebral compliance. Mathematical Medicine and Biology, 25, 113-131. 